\title{
gु
}

\section{Observation of Measurement-Induced Entanglement and Quantum Trajectories of Remote Superconducting Qubits}

\author{
N. Roch, ${ }^{1, *}$ M. E. Schwartz, ${ }^{1}$ F. Motzoi, ${ }^{2}$ C. Macklin, ${ }^{1}$ R. Vijay, ${ }^{3}$ A. W. Eddins, ${ }^{1}$ A. N. Korotkov, ${ }^{4}$ \\ K. B. Whaley, ${ }^{2}$ M. Sarovar, ${ }^{5}$ and I. Siddiqi ${ }^{1}$ \\ ${ }^{1}$ Quantum Nanoelectronics Laboratory, Department of Physics, University of California, Berkeley, California 94720, USA \\ ${ }^{2}$ Department of Chemistry, University of California, Berkeley, California 94720, USA \\ ${ }^{3}$ Department of Condensed Matter Physics and Materials Science, Tata Institute of Fundamental Research, Mumbai 400005, India \\ ${ }^{4}$ Department of Electrical Engineering, University of California, Riverside, California 92521, USA \\ ${ }^{5}$ Scalable and Secure Systems Research (08961), Sandia National Laboratories, Livermore, California 94550, USA
}

(Received 8 February 2014; published 28 April 2014)

\begin{abstract}
The creation of a quantum network requires the distribution of coherent information across macroscopic distances. We demonstrate the entanglement of two superconducting qubits, separated by more than a meter of coaxial cable, by designing a joint measurement that probabilistically projects onto an entangled state. By using a continuous measurement scheme, we are further able to observe single quantum trajectories of the joint two-qubit state, confirming the validity of the quantum Bayesian formalism for a cascaded system. Our results allow us to resolve the dynamics of continuous projection onto the entangled manifold, in quantitative agreement with theory.
\end{abstract}

DOI: 10.1103/PhysRevLett.112.170501

PACS numbers: $03.67 . \mathrm{Bg}, 42.50 . \mathrm{Dv}, 42.50 . \mathrm{Lc}, 85.25 . \mathrm{Dq}$

Entanglement- - the property that binds two independent objects into a single, highly correlated, nonseparable system-is a hallmark of quantum theory. Entanglement schemes for superconducting qubits have traditionally relied on direct qubit-qubit coupling [1,2], cavity-mediated interactions [3], photon-mediated interactions [4], or autonomous cooling [5]. Measurement, in contrast, has traditionally been viewed as a means to restore classical behavior: a quantum system, once observed, is projected onto a single measurement basis state. However, in certain cases, it is possible to design [6-11] a measurement that projects onto an entangled state, thereby purifying, rather than destroying, quantum correlations. Such a measurement has recently been used to entangle two superconducting qubits coupled to the same microwave resonator [12].

Measurement-induced entanglement is a particularly important resource in spatially separated quantum systems, for which no local interactions and therefore no direct methods of creating entanglement exist. Such remote entanglement has been demonstrated using optical photons in several atomic systems [13-15] and nitrogen vacancy centers [16], but has remained elusive for superconducting qubits, which operate in the microwave regime. In this Letter, we demonstrate measurement-induced entanglement between two superconducting qubits, each dispersively [17] coupled to a separate cavity for readout and separated by 1.3 meters of ordinary coaxial cable, by engineering a continuous measurement for which one of the three outcomes is a Bell state [18]. Unlike previous experiments in spatially separated quantum systems, in which the detection of individual spontaneous fluorescence events reveals whether or not entanglement has been generated, we employ time-continuous measurements [19]. This allows us to access the ensemble-averaged dynamics of entanglement generation, which are well described by a statistical model and by a full masterequation treatment. Furthermore, our measurement efficiency is sufficiently high to resolve the individual quantum trajectories in the ensemble [20], thus enabling the observation of the stochastic evolution of a joint two-qubit state under measurement. This functionality sheds new light on the fundamental interplay between entanglement, measurement, and decoherence in a quantum network.

Our experimental apparatus consists of two superconducting transmon qubits placed in spatially separated copper waveguide cavities (three-dimensional transmon architecture) [21]. Each cavity is wound with a superconducting bias coil to enable tuning of the qubit frequency. A weakly coupled port is used for transmission measurements and single qubit control, and a strongly coupled port enables qubit state readout. The strongly coupled ports of the two cavities are connected via two microwave circulators and 1.3 meters of coaxial cable to enable directional transfer of information from cavity 1 to cavity 2 [Fig. 1(a)]. The entire apparatus is contained within an absorptive shield and a Cryoperm magnetic shield to suppress spurious radiation and noise. Qubit and cavity parameters are described in detail in the supplemental information [22].

A joint qubit state measurement can be performed by sequentially driving the cavities in reflection with a nearresonant microwave tone at frequency $\omega_{m}$ that can be described by a classical complex amplitude $\alpha_{\text {in }}$. For a single qubit measured in reflection, the output state is given by $\alpha_{\text {out }}=r^{ \pm} \alpha_{\text {in }}$, where the reflection coefficient $r^{ \pm}$is given by 
(a)

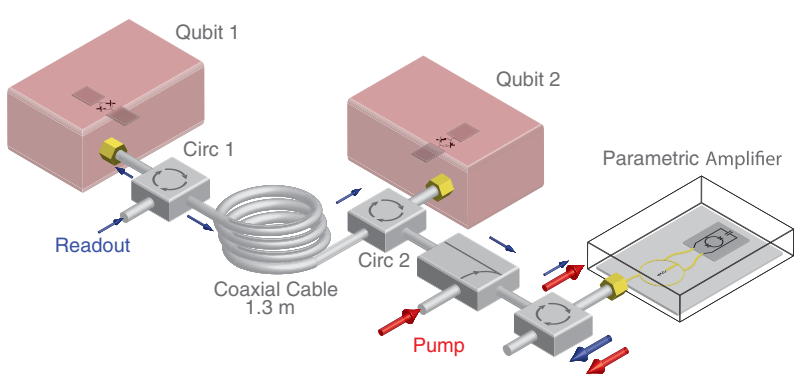

(b) Cavity 1 Output (c) Cavity 2 Output

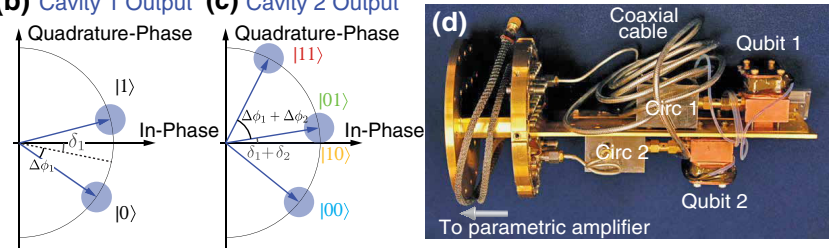

FIG. 1 (color online). Experimental setup. (a) Simplified representation of the experimental setup. (b) and (c) Schematic of the phase shift acquired by a coherent state sequentially measuring first qubit 1 (b) and then qubit 2 (c) in reflection. (d) Picture of the base-temperature setup.

$$
r^{ \pm}=\frac{\kappa-2 i\left(\omega_{r}-\omega_{m} \pm \chi\right)}{\kappa+2 i\left(\omega_{r}-\omega_{m} \pm \chi\right)}
$$

and the signifier $+(-)$ represents the single qubit state $|0\rangle$ $(|1\rangle)$ [22]. Here, $\omega_{r}$ is the bare cavity frequency; $\kappa$ is the cavity decay rate, and $\chi$ is the dispersive shift. The measurement tone acquires a qubit state-dependent phase shift $\phi^{ \pm}=\operatorname{Arg}\left[\alpha_{\text {out }}^{ \pm}\right]$. For the following analysis, it is convenient to define the average and relative phase shifts, $\delta=(1 / 2)\left(\phi^{+}+\phi^{-}\right)$and $\Delta \phi=(1 / 2)\left(\phi^{+}-\phi^{-}\right)$, respectively [see Fig. 1(b)]. For a sequential reflective measurement of two qubits, the output coherent state becomes $\alpha_{\text {out }}=\sqrt{\eta_{\text {loss }}} r_{1}^{ \pm} r_{2}^{ \pm} \alpha_{\text {in }}$, where $\eta_{\text {loss }} \approx 0.81$ represents the efficiency of power transfer between the two cavities. In the general case, $\Delta \phi_{1} \neq \Delta \phi_{2}$ and the phase shifts corresponding to the four basis states $|00\rangle,|01\rangle,|10\rangle$, and $|11\rangle$ are all distinct; the associated measurement decoheres any quantum superposition of states and projects the system into one of the four basis states. However, if we carefully engineer the cavities and the dispersive coupling [22], there exists $\omega_{m}$ such that $\Delta \phi_{1}=\Delta \phi_{2}$. In this situation, the phase shifts associated with states $|01\rangle$ and $|10\rangle$ are identical and equal to $\delta_{1}+\delta_{2}$; the measurement therefore cannot decohere a quantum superposition of $|01\rangle$ and $|10\rangle$ [shown schematically in Fig. 1(c)]. We use a superconducting parametric amplifier [23] to measure the acquired phase shift, realizing a high-fidelity homodyne measurement characterized by a quantum efficiency $\eta_{\text {meas }}=0.4 \pm 0.10$. Figure 2(a) shows a sample time-domain trace of the homodyne signal

$$
V_{m}\left(t_{m}\right)=\frac{1}{t_{m}} \int_{0}^{t_{m}} V(t) d t
$$
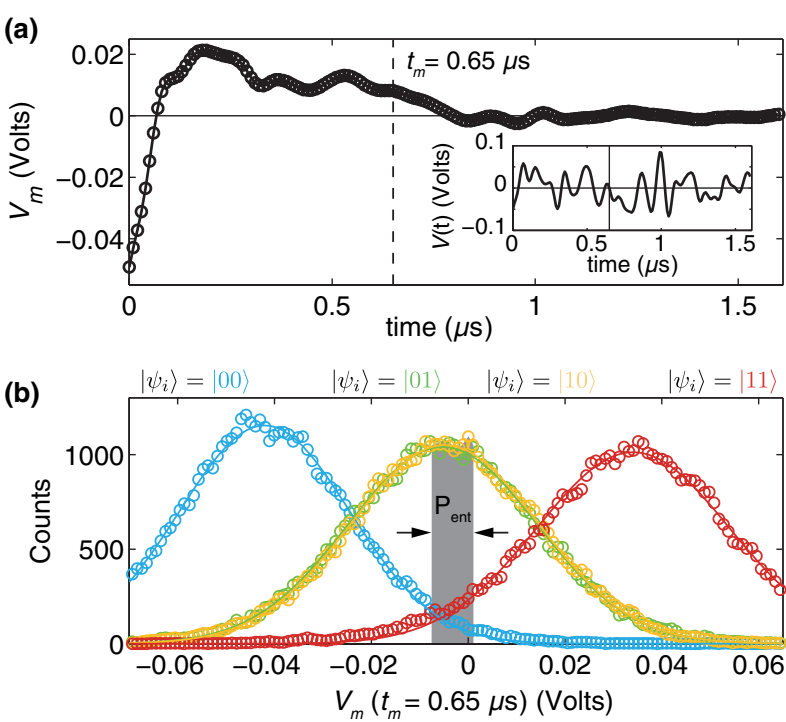

FIG. 2 (color online). Demonstration of indistinguishability between $|01\rangle$ and $|10\rangle$ computational states during measurement. (a) Example of the temporal evolution of the measurement signal $V_{m}$. The inset shows the associated instantaneous voltage $V(t)$. (b) Histogram of $V_{m}$ for each of the four computational states $|00\rangle,|01\rangle,|10\rangle$, and $|11\rangle$. The range of data postselected for tomographic reconstruction at $t_{m}=0.65 \mu \mathrm{s}$ is represented as a shaded grey area.

where $V$ is the instantaneous voltage (inset). We verify that our joint readout cannot distinguish between $|01\rangle$ and $|10\rangle$ by sequentially preparing and then measuring the four basis states. Figure 2(b) represents histograms of $V_{m}$ for a measurement time $t_{m}=0.65 \mu \mathrm{s}$. The states $|00\rangle$ and $|11\rangle$ are well separated, while the histograms for $|01\rangle$ and $|10\rangle$ are fully overlapping, as desired. This enables us to postselect measurement instances that correspond to occupation of the odd-parity manifold without destroying coherence within that manifold and therefore to probabilistically generate entanglement.

We control the rate of entanglement generation

$$
\Gamma_{\text {meas }}=\frac{1}{2} \eta_{\text {meas }} \eta_{\text {loss }}\left|\alpha_{\text {in }}\right|^{2} \sin (2 \Delta \phi)^{2}
$$

by adjusting the measurement strength via the average intracavity photon number $\bar{n}_{1}=(1 / 2)\left(\bar{n}_{1}^{+}+\bar{n}_{1}^{-}\right)$where for each cavity $i[22]$

$$
\bar{n}_{i}^{ \pm}=\frac{\kappa_{i}}{\left(\kappa_{i} / 2\right)^{2}+\left(\omega_{i}-\omega_{m} \pm \chi_{i}\right)^{2}}\left|\alpha_{\mathrm{in}}\right|^{2} .
$$

A photon number $\bar{n}_{1}=1.2$ results in $\Gamma_{\text {meas }} / 2 \pi \approx 210 \mathrm{kHz}$, which sets the characteristic time scale of entanglement generation $\tau_{\text {meas }} \equiv 1 / \Gamma_{\text {meas }} \approx 750 \mathrm{~ns}$. Thus, the dynamics of the measurement process, which are significantly faster than qubit decay rates, can be readily resolved using conventional digital electronics.

To generate and verify entanglement, we implement a sequence of three readout protocols and two qubit rotations. 
We first perform a projective readout $\left(\bar{n}_{1}=6.2\right.$ and $1 \mu \mathrm{s}$ readout length) to postselect the $|00\rangle$ ground state [24]. We then perform $R_{y}^{\pi / 2}$ rotations on both qubits to create the equal superposition state $(1 / 2)(|00\rangle+|01\rangle+|10\rangle+|11\rangle)$. The second readout, which is done in the weak regime and with varying $t_{m}$, stochastically steers the system toward $|00\rangle,|11\rangle$, or the Bell state $(1 / \sqrt{2})(|01\rangle+|10\rangle)$, as documented in the measurement output $V_{m}$. We then apply one of a set of 30 tomographic rotations immediately followed by a strong readout. We repeat this process 8000 times for each tomographic rotation and for each $t_{m}$ to form a single well-averaged data set; we generate an error margin by taking the average and standard deviation of 17 data sets. To produce the density matrix of the postselected entangled state for each time $t_{m}$, we choose an entanglement probability $p_{\text {ent }}$ to constitute the entangled state based on $V_{m}\left(t_{m}\right)$ [shown in the grey shaded region in Fig. 2(b) for $\left.t_{m}=0.65 \mu \mathrm{s}\right]$, and tomographically reconstruct the density matrix using a maximum-likelihood estimator [22]. For perfectly separated histograms, $50 \%$ of the counts will lie in the odd-parity subspace, but we utilize $p_{\text {ent }}=10 \%$ to compensate for imperfect measurement efficiency.

The ability to perform time-continuous measurements enables us to directly observe the ensemble dynamics of the emergence of entanglement. Writing the two-qubit density matrix as $\rho=\sum_{i j k l} \rho_{i j, k l}|i j\rangle\langle k l|$, we can estimate concurrence [25] using the simplified formula [26] $\mathcal{C} \approx$ $\max \left(0,\left|\rho_{01,10}\right|-\sqrt{\rho_{00,00} \rho_{11,11}}\right)$ to characterize the quality of the entanglement during this process. This simplified formula holds when the only nonnegligible off-diagonal elements are $\rho_{01,10}$ and its conjugate, which is applicable to our setup since the high distinguishability between $|00\rangle$, $|11\rangle$ and the $\{|01\rangle,|10\rangle\}$ manifold results in rapid decay of all other off-diagonal elements. Concurrence ranges from zero (for a separable or mixed state) to one (for a maximally entangled two-qubit state) and is greater than zero for all nonseparable two-qubit states [25]. Maximizing $\mathcal{C}$ requires limiting decoherence within the odd-parity manifold, and minimizing stray counts of $|00\rangle$ and $|11\rangle$ by maximizing the signal-to-noise ratio (SNR), defined by the ratio of the separation of the Gaussian histograms [in Fig. 2(b)] to their width, or

$$
\mathrm{SNR} \sim 2\left|\alpha_{\text {in }}\right| \sin (2 \Delta \phi) \sqrt{\eta_{\text {loss }} \eta_{\text {meas }} t_{m}} .
$$

Figure 3 shows the evolution of the concurrence as a function of $t_{m}$. The inset shows the evolution of the relevant density matrix elements (the diagonal elements, representing population probabilities, and the off-diagonal element $\rho_{01,10}$, representing the coherence of the odd-parity subspace).

We note three qualitative regimes: SNR-dominated evolution, stabilization, and decay due to decoherence. Since SNR is proportional to $\sqrt{t_{m}}$, it dominates the evolution at short times $t_{m}<0.75 \tau_{\text {meas }}$. Here, the dynamics are governed by changes to population probabilities, i.e., the increase of $\rho_{01,01}$ and $\rho_{10,10}$ and the decrease of $\rho_{00,00}$ and $\rho_{11,11}$ in the postselected ensemble. The rapid decay of $\rho_{00,00}$ and $\rho_{11,11}$ compared to $\rho_{01,10}$ results in growth of concurrence in this regime. For intermediate times $\left(0.75 \tau_{\text {meas }}<t_{m}<1.25 \tau_{\text {meas }}\right)$, the SNR improvement rate decreases and decoherence begins to take a more noticeable effect. Decoherence is caused by intrinsic dephasing of the qubits $\Gamma_{2, i}^{*}=1 / T_{2, i}^{*}$ and by $\eta_{\text {loss, }}$, which contributes an

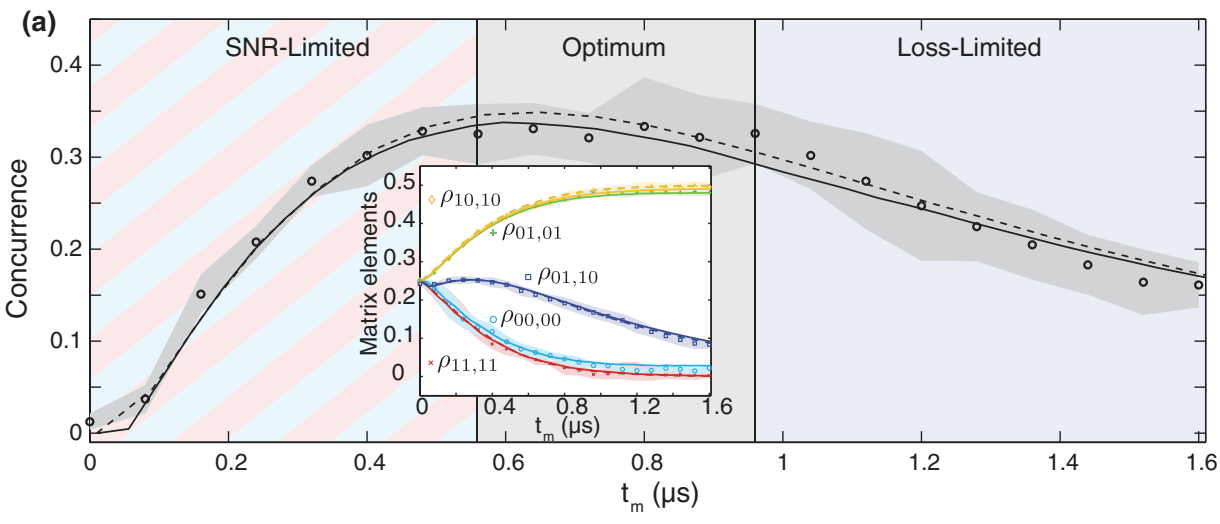

(b)

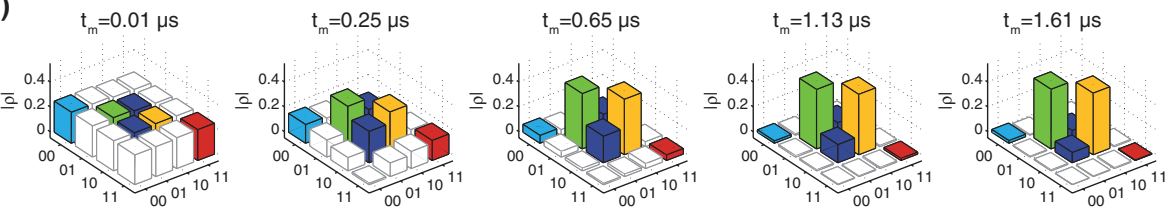

FIG. 3 (color online). Generation and verification of entanglement between two spatially separated superconducting qubits. (a) Concurrence of the entangled state as a function of $t_{m}$. The inset displays the evolution of the basis state populations $\left(\rho_{00,00}\right.$, etc.) and odd-parity coherence $\left(\rho_{01,10}\right)$. The shaded region represents the standard deviation centered about the average (circles). Dashed lines are theoretical simulations based on a Bayesian approach, and solid lines are calculated using a rigorous master equation; in both cases, no fitting parameters are used [22]. (b) Full-density matrices of the postselected entangled subspace for increasing $t_{m}$. 
additional measurement-induced dephasing of the first qubit at a rate

$$
\Gamma_{\text {loss }} \simeq 2\left(1-\eta_{\text {loss }}\right)\left|\alpha_{\text {in }}\right|^{2} \sin (\Delta \phi)^{2} .
$$

At intermediate times, the SNR improvement rate and $\Gamma_{\text {loss }}$ are roughly equal, and hence, the concurrence reaches a maximum value of 0.35 . This value is comparable to what was obtained recently using optical communications $[15,16]$; however, thanks to our time-continuous measurement scheme, the rate at which a qubit-qubit-entangled state is created is orders of magnitude higher $\left(\Gamma_{\text {creation }} /\right.$ $2 \pi=1 \mathrm{kHz})$. For longer times $\left(t_{m}>1.25 \tau_{\text {meas }}\right)$, the density matrix evolution is dominated by decoherence, which eventually drives the system into an incoherent mixture of $|01\rangle$ and $|10\rangle$.

These ensemble dynamics are well described both by a simple statistical model (dashed lines) and by a rigorous master-equation treatment (solid lines) [22]. The models, which account for the chief technical limitations of our scheme (i.e., the inefficiencies $\eta_{\text {loss }}$, the losses between the cavities, and $\eta_{\text {meas }}$, the finite detection efficiency), indicate that reasonable technical improvements could lead to concurrence of $70 \%$, which is comparable to recent single-cavity experiments [12].

Our high-efficiency continuous measurement allows us to go one step further in decomposing the dynamics of measurement-induced entanglement: we can directly observe the individual quantum trajectories [20,27] of our two-qubit system, using a Bayesian update process. In this formalism, $V_{m}(t)$ contains partial quantum state information that allows us to update our estimate of the instantaneous quantum state of the two-qubit system. To validate the Bayesian update for a single trajectory, we generate a mapping $V_{m} \mapsto \rho\left(V_{m}\right)$ : at each time $t_{m}$, we collect all trajectories with similar weak measurement outcomes, $V\left(t_{m}\right)$, and perform a conditional tomographic state reconstruction of those trajectories [see Fig. 4(a)]. We then use this mapping to convert the measured voltage $V_{m}(t)$ for a single experimental realization into $\rho(t)$ and thus reconstruct the quantum trajectory of the system [20]. Figure 4(b) illustrates three typical trajectories, in which the system is projected onto a Bell state or onto the nonentangled states $|00\rangle$ or $|11\rangle$. We see excellent agreement between the tomographic reconstructions of the trajectories and theoretical predictions based on Bayesian updates and a master equation treatment. The observation of these
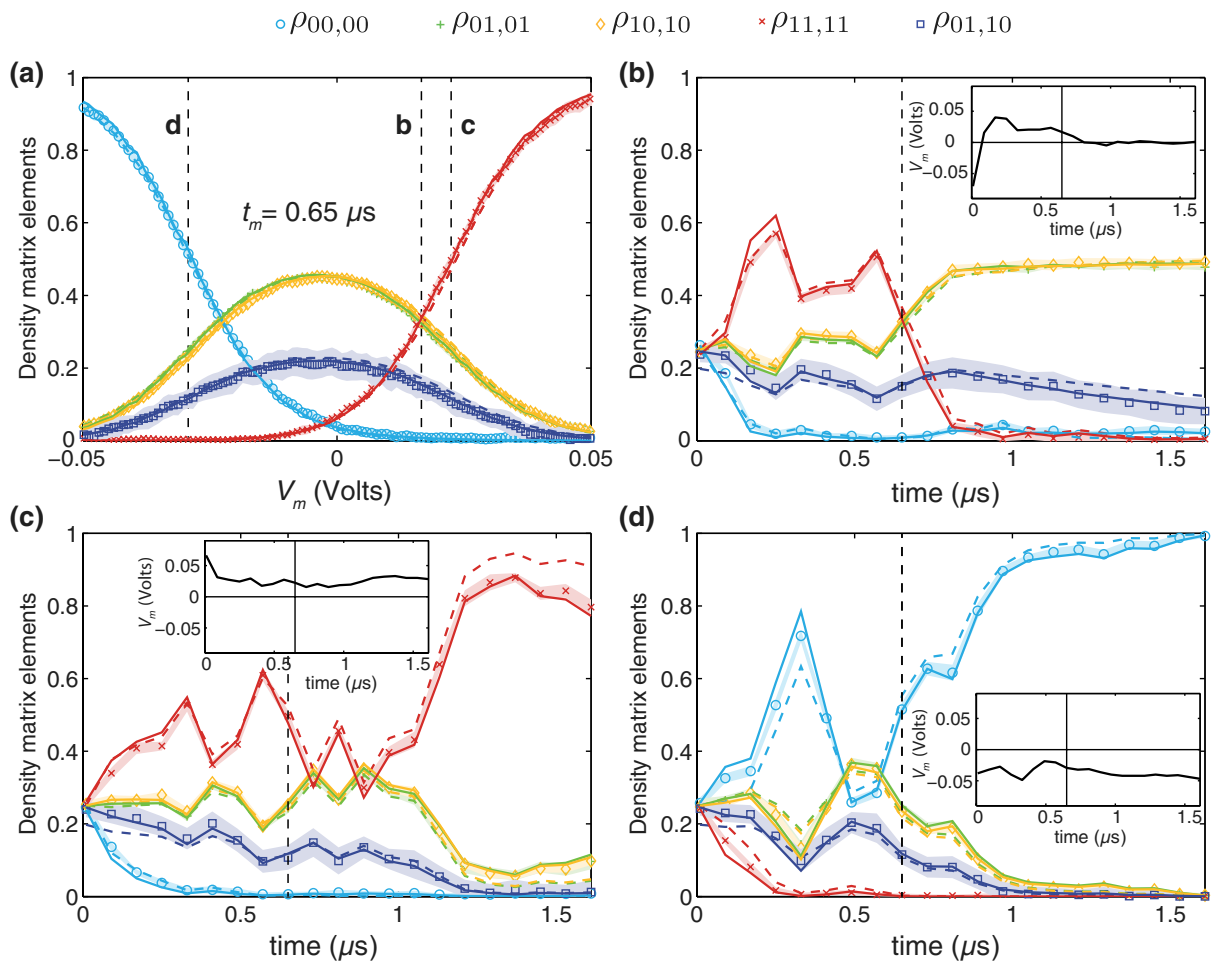

FIG. 4 (color online). Resolving single quantum trajectories for cascaded quantum systems. (a) Absolute value of the density matrix elements conditioned on the measured voltage $V_{m}$ for $t_{m}=0.65 \mu \mathrm{s}$ and $\bar{n}_{1}=1.2$, presenting an instantaneous mapping $V_{m} \mapsto \rho\left(V_{m}\right)$. The shaded region represents the standard deviation about the average (circles); dashed lines (respectively, solid lines) are theoretical simulations based on a Bayesian approach (respectively, on a full master equation) without fitting parameter [22]. (b)-(d) Examples of reconstructed quantum trajectories for diagonal and principal off-diagonal density matrix elements. The dots represent tomographic reconstruction based on the mapping $V_{m} \mapsto \rho\left(V_{m}\right)$ for every $t_{m}$. The dashed lines are Bayesian estimations based on the measured $V_{m}(t)$ (insets). The solid lines for the full master equation were obtained by running 100000 instances of the stochastic differential equation with 1-ns resolution and averaging the obtained populations conditioned on $V_{m}$ at $t_{m}[22]$. 
quantum trajectories shows the novelty and strength of our continuous measurement scheme. Our experiment thus demonstrates the validity of quantum trajectory theories for cascaded quantum systems [28,29], which describe the conditioned evolution of distributed quantum systems.

Our experiments demonstrate that quantum entanglement can be established between distant systems that interact only through a coherent signal propagating along low-loss electrical wires, a functionality that will be integral to the realization of complex, distributed quantum networks. We take advantage of the versatility of continuous measurement to monitor the dynamics of entanglement generation and demonstrate quantitative agreement to a theoretical model that captures the experimental details of the physical circuit [22]. Moreover, our characterization of the state of the joint system under continuous measurement suggests the feasibility of future continuous feedback stabilization of entanglement [30,31]. Further technical improvements in quantum efficiency, coherence times, and transmission characteristics hold the promise of on-demand, stabilized remote entanglement-a powerful resource for quantum information processing.

N. R. and M. E. S. contributed equally to this work. We thank E. M. Levenson-Falk, K. W. Murch, D. H. Slichter, D. M. Toyli, and S. J. Weber for discussions. This research was supported in part by the U.S. Army Research Office, the Intelligence Advanced Research Projects Activity (IARPA). M. E.S. acknowledges support from the Fannie and John Hertz Foundation. R. V. acknowledges support from the Government of India. A. N. K. acknowledges support from ARO under MURI W911NF-11-10268. Sandia National Laboratories is a multiprogram laboratory managed and operated by Sandia Corporation, a wholly owned subsidiary of Lockheed Martin Corporation, for the United States Department of Energy's National Nuclear Security Administration under Contract No. DE-AC04-94AL85000.

"Present address: CNRS and Université Grenoble Alpes, Institut Néel, 38042 Grenoble, France. nicolas.roch@neel.cnrs.fr

[1] M. Steffen, M. Ansmann, R.C. Bialczak, N. Katz, E. Lucero, R. McDermott, M. Neeley, E. M. Weig, A. N. Cleland, and J. M. Martinis, Science 313, 1423 (2006).

[2] A. Dewes, F. R. Ong, V. Schmitt, R. Lauro, N. Boulant, P. Bertet, D. Vion, and D. Esteve, Phys. Rev. Lett. 108, 057002 (2012).

[3] L. DiCarlo, J. M. Chow, J. M. Gambetta, L. S. Bishop, B. R. Johnson, D. I. Schuster, J. Majer, A. Blais, L. Frunzio, S. M. Girvin, and R. J. Schoelkopf, Nature (London) 460, 240 (2009).

[4] A. F. van Loo, A. Fedorov, K. Lalumiére, B. C. Sanders, A. Blais, and A. Wallraff, Science 342, 1494 (2013).

[5] S. Shankar, M. Hatridge, Z. Leghtas, K. M. Sliwa, A. Narla, U. Vool, S. M. Girvin, L. Frunzio, M. Mirrahimi, and M. H. Devoret, Nature (London) 504, 419 (2013).
[6] R. Ruskov and A. N. Korotkov, Phys. Rev. B 67, 241305 (2003).

[7] H.-A. Engel and D. Loss, Science 309, 586 (2005).

[8] B. Trauzettel, A. N. Jordan, C. W. J. Beenakker, and M. Büttiker, Phys. Rev. B 73, 235331 (2006).

[9] C. L. Hutchison, J. M. Gambetta, A. Blais, and F. K. Wilhelm, Can. J. Phys. 87, 225 (2009).

[10] K. Lalumiére, J. M. Gambetta, and A. Blais, Phys. Rev. A 81, 040301 (2010).

[11] F. Helmer and F. Marquardt, Phys. Rev. A 79, 052328 (2009).

[12] D. Risté, M. Dukalski, C. A. Watson, G. de Lange, M. J. Tiggelman, Y. M. Blanter, K. W. Lehnert, R. N. Schouten, and L. DiCarlo, Nature (London) 502, 350 (2013).

[13] C. W. Chou, H. de Riedmatten, D. Felinto, S. V. Polyakov, S. J. van Enk, and H. J. Kimble, Nature (London) 438, 828 (2005).

[14] J. Hofmann, M. Krug, N. Ortegel, L. Gérard, M. Weber, W. Rosenfeld, and H. Weinfurter, Science 337, 72 (2012).

[15] D. L. Moehring, P. Maunz, S. Olmschenk, K. C. Younge, D. N. Matsukevich, L.-M. Duan, and C. Monroe, Nature (London) 449, 68 (2007).

[16] H. Bernien, B. Hensen, W. Pfaff, G. Koolstra, M. S. Blok, L. Robledo, T. H. Taminiau, M. Markham, D. J. Twitchen, L. Childress, and R. Hanson, Nature (London) 497, 86 (2013).

[17] A. Wallraff, D. I. Schuster, A. Blais, L. Frunzio, J. Majer, M. Devoret, S. M. Girvin, and R. J. Schoelkopf, Phys. Rev. Lett. 95, 060501 (2005).

[18] J. Kerckhoff, L. Bouten, A. Silberfarb, and H. Mabuchi, Phys. Rev. A 79 (2009).

[19] M. Hatridge, S. Shankar, M. Mirrahimi, F. Schackert, K. Geerlings, T. Brecht, K. M. Sliwa, B. Abdo, L. Frunzio, S. M. Girvin, R. J. Schoelkopf, and M. H. Devoret, Science 339, 178 (2013).

[20] K. W. Murch, S. J. Weber, C. Macklin, and I. Siddiqi, Nature (London) 502, 211 (2013).

[21] H. Paik, D. I. Schuster, L. S. Bishop, G. Kirchmair, G. Catelani, A. P. Sears, B. R. Johnson, M. J. Reagor, L. Frunzio, L. I. Glazman, S. M. Girvin, M. H. Devoret, and R. J. Schoelkopf, Phys. Rev. Lett. 107, 240501 (2011).

[22] See Supplemental Material at http://link.aps.org/ supplemental/10.1103/PhysRevLett.112.170501 for further information on the theoretical modeling and experimental details.

[23] M. Hatridge, R. Vijay, D. H. Slichter, J. Clarke, and I. Siddiqi, Phys. Rev. B 83, 134501 (2011).

[24] J. E. Johnson, C. Macklin, D. H. Slichter, R. Vijay, E. B. Weingarten, J. Clarke, and I. Siddiqi, Phys. Rev. Lett. 109, 050506 (2012).

[25] W. K. Wootters, Phys. Rev. Lett. 80, 2245 (1998).

[26] L. Jakóbczyk and A. Jamróz, Phys. Lett. A 347, 180 (2005).

[27] P. Campagne-Ibarcq, E. Flurin, N. Roch, D. Darson, P. Morfin, M. Mirrahimi, M. H. Devoret, F. Mallet, and B. Huard, Phys. Rev. X 3, 021008 (2013).

[28] H. J. Carmichael, Phys. Rev. Lett. 70, 2273 (1993).

[29] C. W. Gardiner, Phys. Rev. Lett. 70, 2269 (1993).

[30] M. Sarovar, H.-S. Goan, T. Spiller, and G. Milburn, Phys. Rev. A 72, 062327 (2005).

[31] S. G. Hofer, D. V. Vasilyev, M. Aspelmeyer, and K. Hammerer, Phys. Rev. Lett. 111, 170404 (2013). 